\title{
Reviewer recognition
}

○ International Spinal Cord Society 2019

We would like to thank all those listed below for taking the time to review for Spinal Cord in 2018-your generosity is much appreciated and we hope your association with the journal continues in the future.

Editor-in-Chief: Lisa Harvey

AAbraham, Karen
Ammirati, Enrico
Anderson, Kimberly
Astorino, Todd
Atalay, Basar
Atkinson, Ross
Aubrey, Karin
Baba, Hisatoshi
Ball, Jonathon
Banovac, Kresimir
Barratt, Damien
Bartels, R H
Battistella, Linamara
Baumberger, Michael
Berlowitz, David
Best, Krista-Lynn
Biering-Sørensen, Fin
Bigford, Gregory E.
Birch, Brian
Blight, A
Bolliger, Marc
Bonavita, Jacopo
Bossuyt, Fransiska
Boswell-Ruys, Claire
Brinkhof, Martin
Brock, James
Brooks, Jordan
Brurok, Berit
Bryden, Anne
Bunketorp-Käll, Lina
Bye, Elizabeth
Byra, Stanisława
Calhoun Thielen, Christina
Cameron, Ian
Cao, Yue
Casey, Jackie

Cavalcanti, Geraldo

Chamberlain, Jonviea

Chen, Yuying

Chhabra, Harvinder

Christensen, Peter

Cortes, Mar

Coupaud, Sylvie

Craig, Ashley

Cristante, Alexandre

Culina, Antica

Curt, Armin

De Lure, Federico

De Vries, Wiebe

De Wolf, Annelies

Dehpour, Ahmad reza

Dickson, Hugh

Dijkers, Marcel

Diong, Joanna

DiPiro, Nicole

Divanoglou, Anestis

Donovan, William

Dorsett, Pat

Dorstyn, Diana

Dunlop, Sarah

Eckert, Danny

Elfström, Magnus

Elliott, Stacy

Engkasan, Julia

Erdem, Didem

Erlandsen, Erland

Everaert, Karel

Fattal, Charles

Fehlings, Michael

Ferreiro-Velasco, Malena

Field-Fote, Edelle

Figueiredo Leite, Victor
Finger, Monika

Finlay, Katherine

Flank, Peter

Flueck, Joelle

Fornusek, Che

Fridén, Jan

Frisbie, James

Furlan, Julio

Galea, Mary

Gall, Angela

Gemperli, Armin

Glinsky, Joanne

Gohritz, Andreas

Gomez-Soriano, Julio

Gondim, Francisco

Gorgey, Ashraf

Goto, Takuro

Graco, Marnie

Grassner, Lukas

Gris, Denis

Gross, Mirja

Gruner, John

Gwinn, Tom

Habek, Mario

Hagen, Ellen Merete

Hanci, M

Harel, Noam

Hassett, Leanne

Hatch, Bryndon

Hatton Kolpek, Jimmi

Hayes, Keith

Hearn, Jasmine

Herbert, Robert

Herrero, Azael

Hicks, Audrey

Hofstoetter, Ursula
Hogaboom, Nathan

Hoh, Daniel

Holmes, Gregory

Hong, EunKyoung

Hubli, Michèle

Hughes, Sean

Hwang, Miriam

Illidi, Camilla

Inayama, Takayo

Isaac, Amanda

Jacobs, W B

Jakovcevski, Igor

Janssen, Thomas

Jianjun, Li

Johnson, William

Jorgensen, Sophie

Jorgensen, Vivien

Juneau, Paul

Kalpakjian, Claire

Kanna, Rishi

Kapadia, Naaz

Katoh, Shinsuke

Kendall, Melissa

Kennedy, Gerard

Khoo, Selina

Klekamp, J

Kluger, Patrick

Konstantinos, Margetis

Kramer, John

Krassioukov, Andrei

Krause, James

Krebs, Jorg

Kressler, Jochen

Kumar, Naveen

Kurban, Dilnur

Lane, Michael 
Lavelle, John

Le Fort, Marc

Leblond, Jean

Legge, Michael

Leicht, Christof

Li, Yunbo

Liu, Nan

Lose, G

Lude, Peter

Lynch, John

Macefield, Vaughan

Maciaczyk, Jarek

Mac-Thiong, Jean-Marc

Mailhot, Genevieve

Marino, Ralph

Marklund, Niklas

Marquez, Maria Auxiliadora

Martin Ginis, Kathleen

Mat Rosly, Maziah

McCaughey, Euan

McRae, Jackie

Meade, Michelle

Mehta, Swati

Middleton, James

Migliorini, Christine

Mihara, Hisanori

Misirlioglu, Tugce

Mohammed, Khalid

Monden, Kimberley

Moradi, Nahid

Morishita, Yuichiro

Morris, Norm

Moses, Jenny

Mueller, Gabi

Mulcahey, Mary Jane

Musselman, Kristin

Nakanishi, Kazuyoshi

New, Peter

Nightingale, Tom

Nooijen, Carla

Nout, Yvette

O'Connell, Colleen

O'Hare Doig, Ryan
Okada, Seiji

Oo, WM

Ottomanelli, Lisa

Ozawa, Hiroshi

Ozdemir, Mevci

Pagliacci, Maria

Pan, Shin-Liang

Panisset, Maya

Pannek, Júrgen

Papmeyer, Martina

Paquet, Jerome

Peachment, Ruth

Pelletier, Chelsea

Pennant, William

Pernigotti, Diana

Perret, Claudio

Phillips, Aaron

Piper, Amanda

Pires, Jennifer

Postma, Karin

Previnaire, Jean

Purves, Sheila

Quinzaños, Jimena

Rainer, Abel

Ramer, Matt

Rauch, Alexandra

Redondo-Castro, Elena

Reinhardt, Jan

Rice, Laura

Richard-Denis, Andréane

Richards, J. Scott

Roels, Ellen

Rohn, Edward

Romer, Lee

Ronca, Elias

Ruitenberg, Marc

Rupp, Rüdiger

Rutkowski, Susan

Ryerson Espino, Susan

Saberi, Hooshang

Sabour, Hadis

Sabre, Liis

Sakakibara, Brodie
Salehi-Nejad, Alireza

Sankari, Abdulghani

Scheel-Sailer, Anke

Scheuringer, Monika

Scholz, Martin

Schotland, Helena

Schubert, Martin

Schultke, Elisabeth

Schurr, Karl

Scivoletto, Giorgio

Seel, Ronald

Seif, Maryam

Shabany, Maryam

Shah, Prithvi

Sharif, Hisham

Sharwood, Lisa

Shem, Kazuko

Shin, Hyung Ik

Shokraneh, Farhad

Silveira, Stephanie

Silver, John

Sima, Adam

Sinclair, Andrew

Singal, Raj

Smith, Andrew

Smith, Brett

Smith, Bridget

Smith, Eimear

Soni, Bakul

Stillman, Michael

Stokic, Dobrivoje

Stolwijk-Swuste, Janneke M. Wong, Shun

Suda, Kota

Suzuki, Hidenori

Swaine, Jillian

Sweet, Shane

Tadokoro, Nobuaki

Tajima, Fumihiro

Takahata, Masahiko

Tamburella, Federica

Tanhoffer, Ricardo

Teague, Warwick

Tetzlaff, Wolfram
Tharion, George

Thompson, Floyd

Thumbikat, Pradeep

Totosy de Zepetnek, Julia

Tsitsopoulos, Parmenion

Ushiku, Chikara

Vaidyanathan, Subramanian

Vainrib, Michael

Van de Meent, Henk

Van der Scheer, Jan

Van Diemen, Tijn

Van Zyl, Natasha

Venturelli, Massimo

Vidal Rodriguez, Sonia

Virseda, Miguel

Vosoughi, Fardis

Wadsworth, Brooke

Walter, Matthias

Wangdell, Johanna

Watanabe, Masahiko

Webber, Sandra

Wecht, Jill

Weerts, Eric

Wei, Hong-en

Weidner, Norbert

Welk, Blayne

West, Christopher

Williams, Toni L

Wilson, Jefferson

Wirz, Markus

Wong, Samford

Wood-Wentz, Christina

Wright, Karina

Yang, Jaynie

Yilmaz, Bilge

Zbogar, Dominik

Zeilig, Gabi

Zimmermann, Georg

Zmierczak, Hans Georg 